\title{
Finite-time Convergence Policies in State-dependent Social Networks
}

\author{
Daniel Silvestre, Paulo Rosa, João P. Hespanha, Carlos Silvestre
}

\begin{abstract}
This paper addresses the problem of finite-time convergence in a social network for a political party or an association, modeled as a distributed iterative system with a graph dynamics chosen to mimic how people interact. It is firstly shown that, in this setting, finite-time convergence is achieved only when nodes form a complete network, and that contacting with agents with distinct opinions reduces to a half the required interconnections. Two novel strategies are presented that enable finite-time convergence, even for the case where each node only contacts the two closest neighbors. These strategies are of prime importance, for instance, in a company environment where agents can be motivated to reach faster conclusions. The performance of the proposed policies is assessed through simulation, illustrating, in particular the finite-time convergence property.
\end{abstract}

\section{INTRODUCTION}

The study of social networks relates to understanding how a group of agents perform a decision-making process. We are interested in the problem of showing convergence for a state-dependent social network and what are the implications that small changes on the way the nodes interact can cause in the convergence time. Such observations are interesting in practical terms in the sense that marketing campaigns and proper information dissemination in a network can significantly reduce the convergence time.

In [1], the authors study a classic model of influence networks and opinion formation processes found in sociology, which considers the evolution of power of each agent based on previous opinion formation process outcomes. The focus is on finding how the weights assigned to each agent evolve if they are constructed using previous relevance of a specific node and respective previous weight.

The problem of proving convergence in the presence of leaders is addressed in [2], assuming that the network is statedependent in the sense that nodes communication is based on the difference in state being below a certain threshold of their own. Given that the initial graph has a directed spanning tree, the authors of [2] provide a potential function so as to get the system to consensus while maintaining network connectivity. In this paper, we turn our focus on how to define the statedependent rule to achieve faster convergence, by using an alternative approach, based on recent advances in consensus methods available in the literature.

In [3], it is consider the problem of selecting leaders which are more determinant both to the steady and transient states. Different metric measures for social influence are presented,

\footnotetext{
Contact the author D. Silvestre through dsilvestre@isr.ist.utl.pt for a free unformatted copy of this article or go to http://ieeexplore.ieee.org/abstract/document/7170870/
} for the final formatted version. allowing the construction of a non-convex optimization for the optimal leader selection problem. Convex relaxation techniques are employed and a distributed solution is found. Note that [3] is related to our work since both focus on how fast the agents reach a solution. However, the dynamics assumed in [3] are time-invariant whereas we consider a more general framework.

In this paper, we focus on analyzing a social network within a political party or an association where agents are rational when evaluating each argument of other nodes, and influence occurs among agents whose opinion is closer. The main contributions are as follows:

- We model a social network as a distributed algorithm where the network is state-dependent with a fixed parameter of maximum number of connections;

- Considering only nodes with distinct opinion is shown to reduced the number of required neighbors to half the nodes in the network to obtain finite-time convergence;

- Finally, two strategies are investigated — one where nodes with extreme opinions contact with each other and another where agents require a fixed number of neighbors - and proved to converge in finite time, even when only communicating with 2 other nodes.

The topic of studying convergence of social networks is closely related to that of consensus (see, e.g., [4],[5]). The dynamic system generated has similarities and most tools used in the convergence proofs are common to both fields (see [6], [7], [8], [4] and [5]). The work of [9] addresses the problem of consensus with state-dependent dynamics and the tools to obtain the proofs are similar to those adopted in this paper. Nevertheless, our results focus on how to select the network dynamics to increase the rate of convergence.

The main observation in this paper is that people in social networks such as a political party or a sports association, tend to contact other agents with similar opinions. The work of [10] and [11] points to the same conclusion. Different models are studied in [10], resulting in nodes converging, polarizing or fragmenting into various opinion clusters. In [11], it investigates when randomly selected pairs of nodes average their beliefs, as long as their opinions are close, converge to a single or multiple opinion clusters. In this paper, the main difference is our assumption that each node has a fixed number of influence connections, which is motivated by people having a limited number of acquaintances and considering a small number of agents in their decision.

\section{REFERENCES}

[1] P. Jia, A. Mirtabatabaei, N. E. Friedkin, and F. Bullo, "On the dynamics of influence networks via reflected appraisal," in American 
Control Conference (ACC), 2013, 2013, pp. 1249-1254.

[2] Z. Kan, J. Klotz, E. Pasiliao, and W. Dixon, "Containment control for a directed social network with state-dependent connectivity," in American Control Conference (ACC), 2013, 2013, pp. 1950-1955.

[3] M. Fardad, F. Lin, X. Zhang, and M. Jovanovic, "On new characterizations of social influence in social networks," in American Control Conference (ACC), 2013, June 2013, pp. 4777-4782.

[4] R. Olfati-Saber and R. Murray, "Consensus problems in networks of agents with switching topology and time-delays," Automatic Control, IEEE Transactions on, vol. 49, no. 9, pp. 1520 - 1533, sept. 2004.

[5] F. Bullo, J. Cortés, and S. Martínez, Distributed Control of Robotic Networks, ser. Applied Mathematics Series. Princeton University Press, 2009, electronically available at http://coordinationbook.info.

[6] S. Patterson, B. Bamieh, and A. El Abbadi, "Convergence rates of distributed average consensus with stochastic link failures," Automatic Control, IEEE Transactions on, vol. 55, no. 4, pp. 880 -892, april 2010.

[7] F. Fagnani and S. Zampieri, "Average consensus with packet drop communication," SIAM J. Control Optim, no. 48, pp. 102-133, 2009.

[8] R. Carli, F. Bullo, and S. Zampieri, "Quantized average consensus via dynamic coding/decoding schemes," International Journal of Robust and Nonlinear Control, vol. 20, no. 2, pp. 156-175, 2010. [Online]. Available: http://dx.doi.org/10.1002/rnc.1463

[9] G. Shi and K. H. Johansson, "Convergence of distributed averaging and maximizing algorithms part ii: State-dependent graphs," in American Control Conference (ACC), 2013, 2013, pp. 6875-6880.

[10] R. Hegselmann and U. Krause, "Opinion dynamics and bounded confidence models, analysis and simulation," Journal of Artificial Societies and Social Simulation, vol. 5, no. 3, p. 2, 2002. [Online]. Available: http://ideas.repec.org/a/jas/jasssj/2002-5-2.html

[11] G. Weisbuch, G. Deffuant, F. Amblard, and J.-P. Nadal, "Meet, discuss, and segregate!" Complexity, vol. 7, no. 3, pp. 55-63, 2002. [Online]. Available: http://dx.doi.org/10.1002/cplx.10031 